\title{
Distributed Cooperative Spectrum Sensing based on Weighted Average Consensus
}

\author{
Wenlin Zhang*, Zheng Wang, Yi Guo, Hongbo Liu, Yingying Chen, Joseph Mitola III
}

\begin{abstract}
In this paper, we study the distributed spectrum sensing in cognitive radio networks. Using weighted average consensus algorithm, we develop a weighted soft measurement combining scheme without the centralized fusion center. After the measurement by the energy detector, each secondary user (SU) exchanges their own measurement statistics with its local neighbors, and chooses the information exchanging rate according to the estimated average signal-to-noise ratio (SNR). We prove the convergence of the consensus iteration, and each SU will hold the global decision statistics from the weighted soft measurement combining throughout the network. The proposed scheme is robust with respect to temporary communication link failures. Simulation results show our method has a better performance than the existing average consensus-based approach.
\end{abstract}

\section{INTRODUCTION}

Cognitive Radio (CR) [1] aims to improve the spectrum utilization by allowing unlicensed secondary user (SU) to operate in the 'white spaces' of the licensed spectrum bands. One of the key techniques in cognitive radio is spectrum sensing, which enables the secondary users to detect the absence or presence of the primary user (PU) in the spectrum. Recent research progress shows cooperative spectrum sensing is an effective and promising methodology to improve the spectrum sensing performance under practical shadowing, fading and time-varying wireless channels [2]. In this paper, we consider the weighted average consensus-based approach for distributed cooperative spectrum sensing.

The main advantage of cooperative spectrum sensing is to enhance the sensing performance by exploiting the observation diversity of the spatially located SUs. By cooperation, $\mathrm{CR}$ users can share their sensing information for making a combined decision which is more accurate than the individual decisions [2]. Among many works on cooperative spectrum sensing, most studies focused on developing centralized cooperation approaches [3]. There are two stages in the cooperative sensing. In the first stage, each SU makes the measurement using appropriate detecting techniques. In the second stage, SUs report their measurement information to the centralized fusion center, then a global decision is made at the fusion center according to certain measurement combining method.

In the sensing stage, energy detection is widely applied because it requires lower design complexity and no priori knowledge of primary users, compared to other techniques,

W. Zhang, Y. Guo, H. Liu, Y. Chen, J. Mitola are with the Electrical \& Computer Engineering Dept., Stevens Institute of Technology, Hoboken, NJ 07030, USA. Z. Wang is with the Electrical \& Computer Engineering Dept., University of Toronto, Toronto, ON, M5S 3G4, Canada.

* Corresponding author, email: wzhang5@stevens.edu (W. Zhang) such as matched filter detection or cyclostationary detection [4]. During fusion, different measurement combining methods have been considered including hard bit combining [5], soft gain combining [6][7]. However, centralized cooperative spectrum sensing requires the entire received data be gathered at one place. In large SU networks, it is less scalable, less fault tolerant, and has redundant reporting cost. To overcome these disadvantages, bio-inspired consensusbased scheme is introduced to spectrum sensing in [8] for the decentralized measurement combining for the cooperative SUs. However, average consensus only ensures the average soft combining of the global measurements, which is less effective under the practical Rayleigh fading channels.

The main contribution of this paper is to propose a weighted soft measurement combining scheme without the centralized fusion center. After the measurement by the energy detector, SUs exchange their decision statistics with their local neighbors, and weight the information exchanging rate according to their own estimated average signal-tonoise ration (SNR) values. We rigorously prove that after the consensus is reached, each SU holds the global decision statistics from the weighted soft combining throughout the network. The convergence of the algorithm will not be affected by the communication link switching or temporary link failures. The simulation results show the significant improvement of the sensing performance compared to the existing consensus-based approach, especially under the low SNR Rayleigh fading channels.

The rest of this paper is organized as follows: In section II, we introduce the fundamentals about the energy detector and the consensus related SU network model. We describe our proposed weighted average consensus-based spectrum sensing scheme in Section III. In section IV, we present simulation results and make comparison with the existing approach. We conclude this study in Section V. The related theoretic proofs are given in the Appendix.

\section{Spectrum Sensing And Secondary Users NETWORK MODELING}

In the consensus-based spectrum sensing scenario, a distributed network is established by the SU group. The related models on the sensing stage and information fusion stage are presented in this section.

\section{A. Sensing and Measurement Stage}

In this paper, we mainly consider the energy detector under the Rayleigh fading channel model [9]. For each SU, the 
received signal $y(t)$ is modeled as

$$
y(t)=\left\{\begin{array}{cc}
n(t), & H_{0} \\
h x(t)+n(t), & H_{1},
\end{array}\right.
$$

where $h$ represents gain of the channel, $x(t)$ is the signal from PU, $n(t)$ is the additive white Gaussian noise.

Following [9][10], under Rayleigh fading, the channel gain $h$ is random and the resulting SNR $\gamma$ follows an exponential distribution. The output $Y$ of each energy detector has the following distributions under hypothesis:

$$
Y \sim\left\{\begin{array}{cc}
\chi_{2 T W}^{2}, & H_{0} \\
\chi_{2 T W-2}^{2}+Y_{e}, & H_{1},
\end{array}\right.
$$

where $\chi_{2 T W}^{2}$ and $\chi_{2 T W-2}^{2}$ denote random quantities under central chi-square distributions with mean $2 T W$ and $2 T W-2$, respectively. $Y_{e}$ has an exponential distribution with parameter $2(\bar{\gamma}+1), \bar{\gamma}$ represents the average SNR of the fading channel. The hypothesis $H_{0}$ and $H_{1}$ denote the absence and presence of the primary user, respectively. $T W$ is the preset time-bandwidth product of the energy detector which can be set to any integer, we denote $T W=m$ for simplicity.

In the consensus-based scenario, we assume $n$ SUs cooperate with each other. The $i^{t h} \mathrm{SU}$ detects the energy and obtain the measurement $Y_{i} \in \mathbb{R}^{+}$. Since the distributions under $H_{0}$ and $H_{1}$ hold the mean $2 m$ and $2 m+2 \bar{\gamma}$, respectively, each SU can simply estimates the average SNR from recent measurements as [7]

$$
\bar{\gamma}_{i}=\frac{1}{2 l} \sum_{j=k-l}^{k}\left(Y_{i, j}-2 m\right)
$$

where $Y_{i, j}$ is the $j^{t h}$ measurement of the $i^{t h}$ secondary user, $l$ is the estimation window.

\section{B. The SU Network Model and Consensus Notions}

In the information fusion stage, SUs communicate with their local neighbors through the SU network and adopt the consensus iteration to obtain the global measurement statistics. For convenience, we assign an index set $\mathcal{I}=$ $\{1,2, \ldots, n\}$ for the SU network formed by $n$ SUs.

To model the consensus algorithm, we adopt the standard undirected graph model for the bidirectional SU communication network. The SU network is represented by an undirected graph $\mathcal{G}=(\mathcal{E}, \mathcal{V})$, where $\mathcal{V}=\left\{v_{i} \mid i \in \mathcal{I}\right\}$ is a finite nonempty set of nodes. We refer the $i^{\text {th }}$ node as the $i^{\text {th }}$ SU. The two names, SU and node, will be used alternatively. The edge set $\mathcal{E}=\left\{e_{i j}=\left(v_{i}, v_{j}\right) \mid i, j \in \mathcal{I}\right\}$. The set of neighbors of node $i$ is denoted by $\mathcal{N}_{i}=\left\{j: e_{i j} \in \mathcal{E}\right\}$. A path in $\mathcal{G}$ consists of a sequence of nodes $v_{1}, v_{2}, \ldots, v_{l}, l \geq 2$, satisfying $\left(e_{m, m+1}\right) \in \mathcal{E}, \forall 1 \leq m \leq l-1$. The graph $\mathcal{G}$ is connected if any two distinct nodes in $\mathcal{G}$ are connected by a path. When considering the directed graph (i.e. digraph), we refer to $v_{i}$ and $v_{j}$ as the tail and head of a directed edge $e_{i j}=$ $\left(v_{i}, v_{j}\right)$, which represents the unidirectional communication link between two neighboring SUs. A digraph is called strongly connected if it is possible to reach any node starting from any other node following the edge directions.
In the case of the time-varying communication links, we model the SU network by $\mathcal{G}(k)=(\mathcal{E}(k), \mathcal{V})$, where $\mathcal{E}(t)$ is the set of active edges at time $k$. Let $\mathcal{N}_{i}(k)=\{j \in \mathcal{V} \mid\{i, j \in$ $\mathcal{E}(k)\}\}$, and $d_{i}(k)=\mathcal{N}_{i}(k)$ denote the degree (number of neighbors) of node $i$ at time $k$.

Let $\mathcal{G}_{i}=\left(\mathcal{E}_{i}, \mathcal{V}\right), i=1, \ldots, r$, denote a finite collection of graphs with common vertex set $\mathcal{V}$. Their union is a graph $\mathcal{G}$ with the same vertex set and an edge set that is the union of the $\mathcal{E}_{i}^{\prime} s$, i.e., $\mathcal{G}=\bigcup_{i=1}^{r} \mathcal{G}_{i}=\left(\bigcup_{i=1}^{r} \mathcal{E}_{i}, \mathcal{V}\right)$. The set of undirected graphs $\left\{\mathcal{G}_{1}, \ldots, \mathcal{G}_{r}\right\}$ is called jointly connected if their union is a connected graph. The set of digraphs $\left\{\mathcal{G}_{1}, \ldots, \mathcal{G}_{r}\right\}$ is called jointly connected if their union is a strongly connected digraph.

In the consensus-based spectrum sensing context, for the $n$ SUs modeled by the graph $\mathcal{G}$, the $i^{\text {th }} \mathrm{SU}$ is assigned a state variable $x_{i}, i \in \mathcal{I}$. The $i^{t h} \mathrm{SU}$ uses $x_{i}$ for representing its measurement statistics of the energy detection. By reaching consensus, we mean the individual state $x_{i}$ asymptotically converge to a common value $x^{*}$, i.e.,

$$
x_{i}(k) \rightarrow x^{*} \text { as } k \rightarrow \infty, \forall i \in \mathcal{I},
$$

where $k$ is the discrete time step, $k=0,1,2, \ldots$, and $x_{i}(k)$ is updated based on the previous states of node $i$ and its neighbors.

\section{Spectrum Sensing BASED ON Weighted Average Consensus}

In this section, we present consensus-based distributed scheme to achieve the weighted measurement combining through local interactions among secondary users, in stead of processing the measurements in the centralized fusion center.

\section{A. Weighted Average Consensus Algorithm}

Following the notation of consensus [11], we denote the value of the $i^{\text {th }}$ agent's measurement $Y_{i}$ as $x_{i}$, and the proposed weighted average consensus-based combining scheme is shown as

$$
x_{i}(k+1)=x_{i}(k)+\frac{\epsilon}{\sigma_{i}} \sum_{j \in N_{i}}\left(x_{j}(k)-x_{i}(k)\right), i \in \mathcal{I}
$$

where $\epsilon$ is the iteration step size satisfying the maximum node degree constraint [11], and $\sigma_{i} \geq 1$ is the weighting ratio.

Remark 1: An important feature of the algorithm is the choice of the weighting factor $\sigma_{i}$. If the $i^{\text {th }} \mathrm{SU}$ has higher confidence about its own measurement, it sets a larger $\sigma_{i}$, which makes the iteration (5) rely less on the local information exchange. On the contrary, an SU with lower measurement confidence will set a smaller $\sigma_{i}$ and relies more on the information from the network in order to improve the overall performance. Therefore, the statistics value across the whole SU network will be dominated by the SUs with higher confidence.

Next, we show that if the communication topologies formed by the SU network are jointly connected, all the 
SUs' decision statistics will reach consensus. The final convergence value is:

$$
x_{i}(k) \rightarrow x^{*}=\frac{\sum_{i=1}^{n} \sigma_{i} x_{i}(0)}{\sum_{i=1}^{n} \sigma_{i}} \text { as } k \rightarrow \infty, \forall i \in \mathcal{I} .
$$

\section{B. Weighted Measurement Combining}

In cooperative spectrum sensing, weighted combining is a natural scheme to improve the detecting performance [6][7]. The global decision statistic $Y_{g}$ is from the weighted sum of the $n$ local measurements

$$
Y_{g}=\sum_{i=1}^{n} \omega_{i} Y_{i}
$$

where $Y_{i}$ is the non-quantized output from the energy detector of the $i^{t h}$ secondary user, and $\omega_{i}$ is the related weight. Particularly, when $\omega_{i}=1 / n, \forall i \in \mathcal{I}, Y_{g}$ is the average of the global measurements.

In [8], an average consensus-based approach is proposed to ensure the distributed measurement combining. In detail, the fusion algorithm is shown as

$$
x_{i}(k+1)=x_{i}(k)+\epsilon \sum_{j \in N_{i}}\left(x_{j}(k)-x_{i}(k)\right), i \in \mathcal{I}
$$

where $\epsilon$ satisfies the maximum node degree constraint [11]. After the algorithm converges, the average measurement combining is achieved as

$$
x_{i}(k) \rightarrow x^{*}=\frac{\sum_{i=1}^{n} x_{i}(0)}{n} \text { as } k \rightarrow \infty, \forall i \in \mathcal{I} .
$$

We refer this case as equal gain combining ( $E G C$ ). Apparently, the $E G C$ approach does not consider the practical channel conditions and the channel fading will affect its performance.

In contrast, the proposed fusion algorithm (5) aims to take each SU's channel condition into consideration. To account for the Rayleigh fading effects, a simple method is setting the weight $\omega_{i}$ directly from the estimated average SNR as [7]

$$
\omega_{i}=\frac{\bar{\gamma}_{i}}{\sum_{i=1}^{N} \bar{\gamma}_{i}}
$$

Accordingly, if we choose the ratio $\sigma_{i}$ in (5) as

$$
\sigma_{i}=\left\{\begin{array}{cc}
\bar{\gamma}_{i}, & \text { if } \bar{\gamma}_{i} \geq 1 \\
1, & \text { if } \bar{\gamma}_{i}<1
\end{array}\right.
$$

where $\bar{\gamma}_{i}$ is obtained from (3), and the saturation is to ensure $\sigma_{i} \geq 1$ for all $i$, then the decision value (6) of the consensus algorithm equals to

$$
x_{i} \rightarrow x^{*}=\frac{\sum_{i=1}^{n} \bar{\gamma}_{i} x_{i}(0)}{\sum_{i=1}^{n} \bar{\gamma}_{i}} .
$$

The sensing performance according to the weighted combining (12) is intended to outperform the performance of the $E G C$ approach under Rayleigh fading channels. We refer our proposed method as weighted gain combining (WGC).
Finally, by comparing the decision value $x^{*}$ with a predefined threshold $\lambda$, every SU locally obtains the global decision as:

$$
\text { Decision } H=\left\{\begin{array}{cc}
1, & x_{i}=x^{*}>\lambda \\
0, & \text { otherwise. }
\end{array}\right.
$$

\section{The Main Theorem}

For convenience, we re-write the algorithm (5) in the following compact form:

$$
x(k+1)=P x(k),
$$

where $x=\left[x_{1}, \ldots, x_{n}\right]^{T}$, and $P$ is the so-called Perron matrix [11], defined as

$$
P=I-\epsilon \Sigma^{-1} L
$$

where $\Sigma=\operatorname{diag}\left\{\sigma_{1}, \ldots, \sigma_{n}\right\}, L \in \mathbb{R}^{n \times n}$ is the Laplacian matrix of the communication graph $\mathcal{G}$ formed by the secondary user nodes, chosen as

$$
l_{i j}=\left\{\begin{array}{cl}
d_{i}, & \text { if } i=j, \\
-1, & \text { if } i \neq j, \\
0, & \text { otherwise, }
\end{array} \quad j \in \mathcal{N}_{i},\right.
$$

where $d_{i}=\left|\mathcal{N}_{i}\right|$ is the degree of node $i$. The maximum node degree constraint [11] means

$$
0<\epsilon<\left(\max _{i}\left|\mathcal{N}_{i}\right|\right)^{-1} \triangleq \frac{1}{\Delta}, i \in \mathcal{I}
$$

where $\Delta$ is called the maximum node degree of the network.

For a network of $n$ secondary users, there are a finite number, say a total of $r$, of possible communication graphs. We denote the set of all possible graphs by $\left\{G_{1}, \ldots, G_{r}\right\}$, and the set of corresponding Perron matrices by $\left\{P_{1}, \ldots, P_{r}\right\}$. The weighted average consensus algorithm is given by

$$
x(k+1)=P_{i(k)} x(k),
$$

where the indices $i(k)$ are integers and satisfy $1 \leq i(k) \leq r$ for all $k>0$.

Theorem 1: For the iteration process (18), if the step size $\epsilon$ satisfies maximum node degree constraint, and the elements of matrix $\Sigma=\operatorname{diag}\left\{\sigma_{1}, \ldots, \sigma_{n}\right\}$ satisfy $\sigma_{i} \geq 1, \forall i \in \mathcal{I}$, and the collection of bidirectional communication graphs that occur infinitely often are jointly connected, then the iteration converges as

$$
\lim _{k \rightarrow \infty} P_{i(k)}^{k} x(0)=\frac{\sum_{i=1}^{n} \sigma_{i} x_{i}(0)}{\sum_{i=1}^{n} \sigma_{i}} \mathbf{1} .
$$

That is, the algorithm (5) converges to:

$$
\lim _{k \rightarrow \infty} x_{i}(k)=\frac{\sum_{i=1}^{n} \sigma_{i} x_{i}(0)}{\sum_{i=1}^{n} \sigma_{i}}, \forall i \in \mathcal{I} .
$$

The proof is given in Appendix A.

Remark 2: Theorem 1 requires weak long-term connectivity which contains both deterministic and stochastic timevarying graph sequences, and the convergence rate in general may not exist. If we further assume each link has an independent probability to fail, then follow the work of [12], 
the algorithm will converge with probability 1 . The variance of total deviation from weighted average is given as

$$
E\left(\left\|x(k)-\frac{\sum_{i=1}^{n} \sigma_{i} x_{i}(0)}{\sum_{i=1}^{n} \sigma_{i}} \mathbf{1}\right\|^{2}\right) \rightarrow 0 \text { as } k \rightarrow \infty,
$$

and the convergence rate is exponential.

Remark 3: Theorem 1 encompasses the average consensus in [13] as a special case when $\Sigma=I$ and $P_{i(k)}$ are symmetric matrices. Apparently, fixed communication topology is a special case when $P_{i(k)}=P, \forall k \geq 0$, where the convergence rate is also exponential given by the PerronFrobenius Theorem [14].

With the maximum node degree weights setup, we can further extend the results to unidirectional communication links assuming the communication topologies are balanced graphs, that is, in every iteration step, the number of neighbors each SU receives information from is the same as the number of neighbors to which it sends out information.

Corollary 1: For the iteration process (18), if the communication topology of the SU network is a balanced graph in every iteration step, and the collection of unidirectional communication graphs that occur infinitely often are jointly connected, then under the same conditions of Theorem 1 , the iteration converges to

$$
\lim _{k \rightarrow \infty} P_{i(k)}^{k} x(0)=\frac{\sum_{i=1}^{n} \sigma_{i} x_{i}(0)}{\sum_{i=1}^{n} \sigma_{i}} \mathbf{1}
$$

The proof is given in Appendix B.

Remark 4: In the iteration, maintaining node degree balanced is a distributed constraint for each SU, without centralized information.

\section{Simulations and Discussions}

We conducted simulations to evaluate the effectiveness of our scheme. For the performance evaluation, we adopt the Monte Carlo method since closed form solutions for detection probability and false alarm do not exist generally.

\section{A. Network Setup}

To compare with the existing consensus-based scheme, we set up a similar scenario as in [8]. In particular, 10 SUs cooperate with each other and form a communication graph as shown in Fig. 1. All SUs are assumed to be static and experiencing i.i.d. Rayleigh fading without spacial correlation, and they are running the synchronized clock during the sensing process and the consensus iteration.

In the sensing stage, we directly generate the output $Y_{i}$ of every SU's energy detector individually under the hypothesis $H_{1}$ in (2), with $T W=6$ at the selected center frequency and bandwidth of interest. Each SU estimates the average SNR $\bar{\gamma}_{i}$ from (3) with the estimation window $l=2$. Each SU then sets $x_{i}(0)=Y_{i}$ and starts the measurement fusion using the proposed scheme with the step size $\epsilon=0.19$ in Eqn. (5). The final decision is made after the consensus result $x^{*}$ is reached.

Fig. 2 shows the convergence of the proposed algorithm iteration under fixed communication graph. We found that

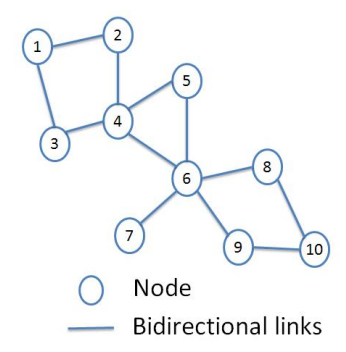

(a) Fixed graph

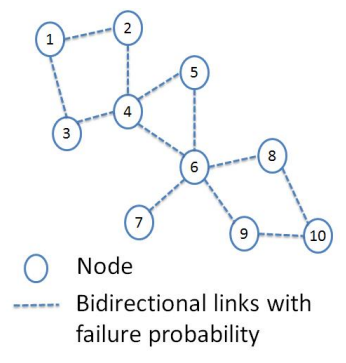

(b) Random graph
Fig. 1. Communication network of 10 SUs [8].

within 30 steps the difference of $x_{i}$ among the SU network are less than $1 \mathrm{~dB}$, which indicates the consensus has been reached on the global decision statistics. Fig. 3 shows the convergence of the algorithm under random graph process with independent link failures. We set the failure probability of each link to 0.4. We observed that the consensus is achieved within 40 iteration steps, which is slightly slower than the fixed graph case.

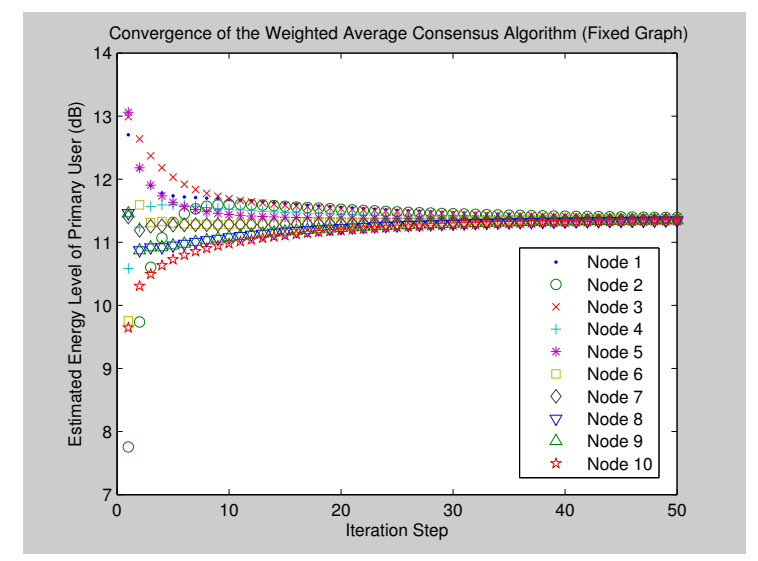

Fig. 2. Convergence of the network with a 10-node fixed graph. $\epsilon=0.19$

Under the bidirectional communication link condition, for fixed topology, shown in Fig. 1(a), we denote our results as Weighted Gain Combining ( $W G C$ ), and denote the results in [8] as Equal Gain Combining $(E G C)$. When the communication links have independent probability of $40 \%$ to fail, as shown in Fig. 1(b), we denote simulation results as $W G C_{s}$ and $E G C_{s}$, respectively.

\section{B. Metrics}

For comparison, we mainly consider $P_{d}$ (probability of detection), $P_{f}$ (probability of false alarm), and average SNR $\gamma$ as metrics. A high $P_{d}$ will result in high $P_{f}$, which increases the interference to primary users. On the other hand, a low $P_{f}$ will result in low $P_{d}$ and lead to low spectrum utilization. The average SNR in the measuring channel reflects the channel condition. It is challenging to 


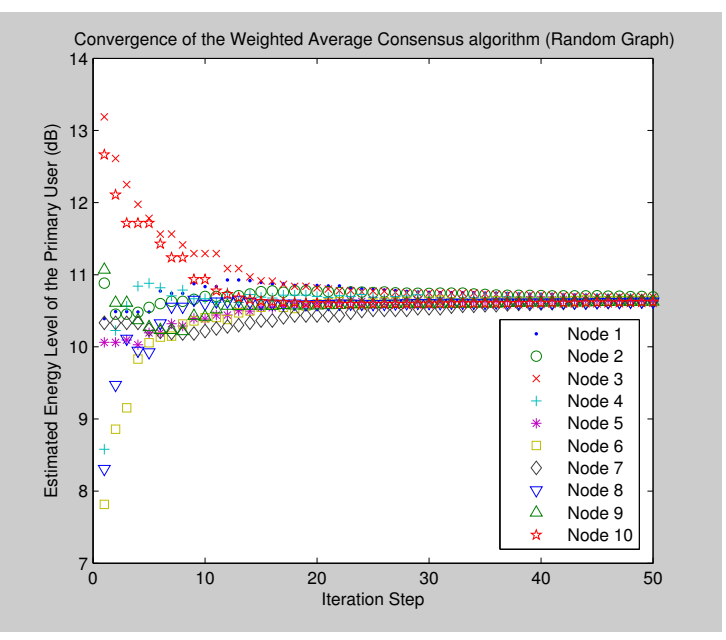

Fig. 3. Convergence of the network with a 10-node random graph, $\epsilon=$ 0.19 . The convergence failure probability is 0 .

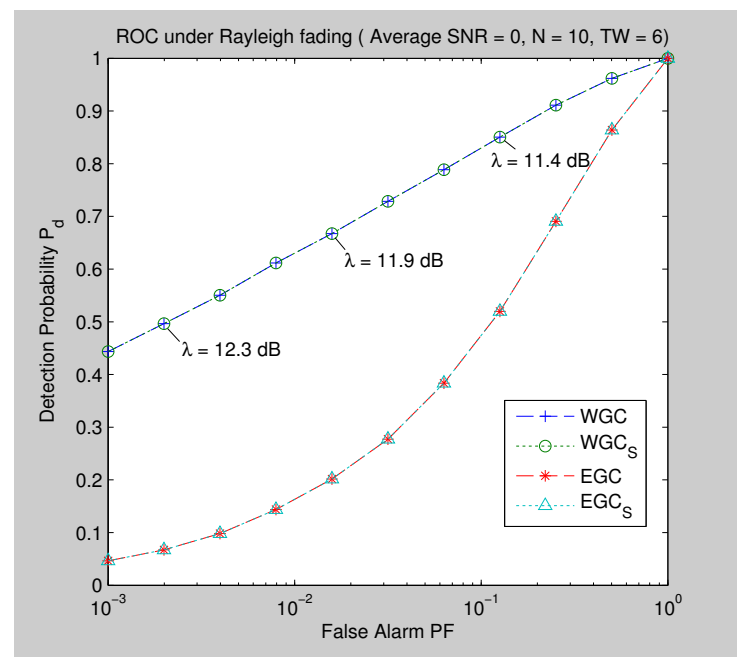

Fig. 4. Receiver operating characteristics under i.i.d Rayleigh fading (Each secondary user has the same average SNR, $\bar{\gamma}_{i}=0 \mathrm{~dB}$ ).

achieve high detecting performances under the condition of low average SNR.

The threshold $\lambda$ is computed from the false alarm constraints and the hypothesis $H_{0}$ in (2). The two schemes share the same threshold, since the measurement output under $H_{0}$ is independent of the average SNR when the primary user signal is absent.

\section{Performance Evaluation}

Fig. 4 shows the receiver operating characteristics (ROC) of the two schemes when the average SNR is OdB in each SU's measurement. We can see clearly from this figure, average consensus-based scheme ( $E G C$ and $\left.E G C_{s}\right)$ does not perform well in this case and the performance has been significantly improved by our proposed weighted average consensus scheme ( $W G C$ and $W G C_{s}$ ).

Fig. 5 shows the ROC of the two schemes when the average SNR of the SU's measurements vary from $0 \mathrm{~dB}$ to
$5 \mathrm{~dB}$. The proposed weighted combining scheme ( $W G C$ and $W G C_{s}$ ) achieves a satisfactory performance. When the false alarm is set to $10^{-2}$, the detection probability reaches 0.8 , while the equal gain scheme ( $E G C$ and $E G C_{s}$ ) is still below 0.5 .

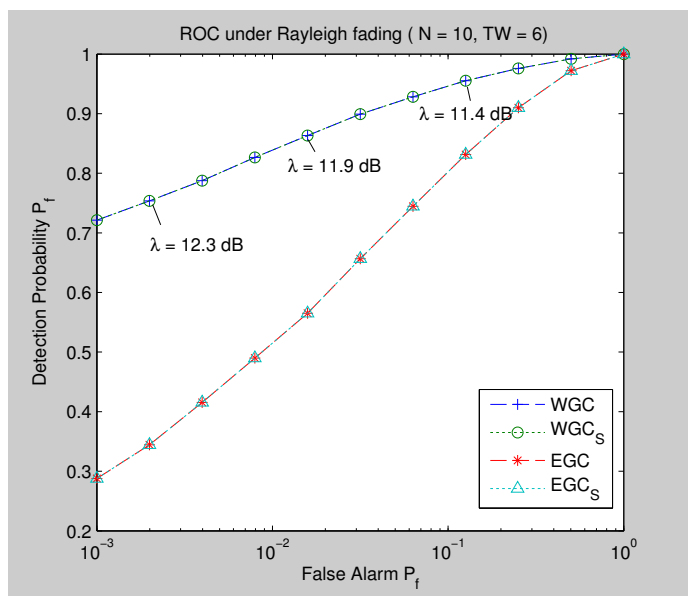

Fig. 5. Receiver operating characteristics under i.i.d Rayleigh fading (Each secondary user has different average SNR, $\bar{\gamma}=[5,1,2,3,4,0,4,3,2,5]$ ).

Fig. 6 shows the detection probability curves with respect to the average SNR when the false alarm probability constraint is 0.005 . We can see that when the average SNR is below $6 \mathrm{~dB}$, our proposed scheme ( $W G C$ and $W G C_{s}$ ) achieves much better performance than the equal gain combining ( $E G C$ and $E G C_{s}$ ). When the average SNR is higher than $6 \mathrm{~dB}$, the performance of these two schemes becomes closer, however, the weighted combining ( $W G C$ and $W G C_{s}$ ) still achieves higher detection probability.

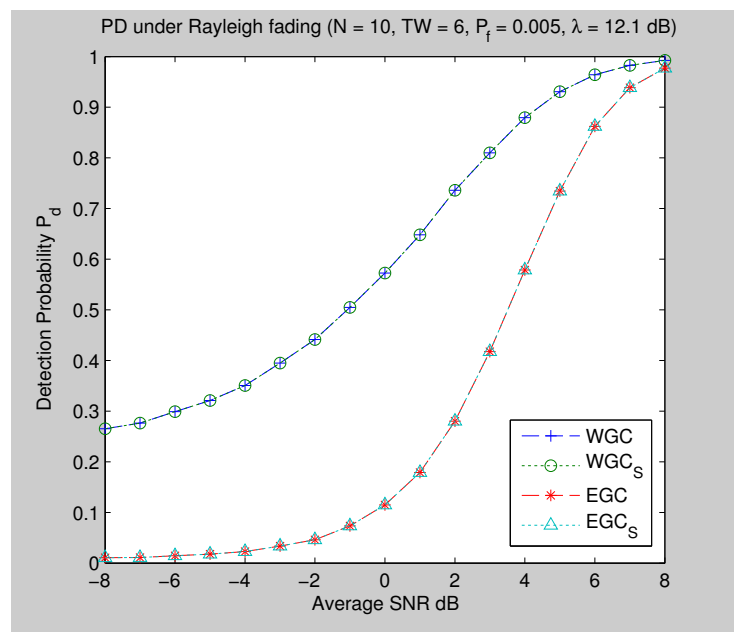

Fig. 6. Detection probability $\left(P_{d}\right)$ with respect to average SNR under i.i.d. Rayleigh fading when the false alarm probability constraint is $P_{f}=0.005$.

\section{CONCLUSION AND FUtURE WORK}

In this paper, we present a distributed weighted combining scheme for the cooperative spectrum sensing in cognitive radio networks. The proposed method is based on the weighted 
average consensus algorithm for both fixed and time-varying graphs. Through weighted local fusion iteration, each SU holds the global decision statistic from the weighted soft measurement combining throughout the network. Simulation results show our approach outperforms the existing average consensus-based equal gain combining scheme under low SNR measurement conditions. Future work will pursue distributed asynchronous sensing and communciation.

\section{ACKNOWLEDGMENT}

Wenlin Zhang, Zheng Wang, and Yi Guo would like to acknowledge the support by the National Science Foundation under Grant EFRI-1024660. Hongbo Liu and Yingying Chen would like to acknowledge the support by the National Science Foundation under Grant CNS-0954020.

\section{APPENDIX}

\section{A. Proof of Theorem 1}

Proof of Theorem 1 shows that consensus iteration (18) actually is a paracontraction process and its fixed point is decided by the eigenspaces of the related Perron matrices.

For a connected undirected graph $\mathcal{G}$ and the associated Perron matrix $P=I-\epsilon \Sigma^{-1} L$ defined in (15), if we see each undirected link of the graph $\mathcal{G}$ as two directed links with opposite directions and different weights, then $\hat{L}=\Sigma^{-1} L$ is the Laplacian matrix of a strongly connected digraph $\hat{\mathcal{G}}$, and $P$ is a nonnegative primitive matrix [11] having 1 as the right eigenvector associated with the eigenvalue 1 . According to [11], $P$ has the eigenvalue 1 as the maximum modulus, and all the other eigenvalues are in the unit circle, i.e., $\left|\lambda_{i}\right|<$ $1, i=2, \ldots, n$. Therefore, we have $\|P x\| \leq\|P\|\|x\|=\|$ $x \|, \forall x \in \mathbb{R}^{n}$, which proves $P$ is a paracontracting matrix [15]. For a paracontracting matrix $M$, we denote $\mathcal{H}(M)$ as its fixed-point subspace, i.e., $\mathcal{H}(M)=\left\{x\left|x \in \mathbb{R}^{n}\right| M x=x\right\}$. Apparently, $\mathcal{H}(M)$ is $M$ 's eigenspace associated with the eigenvalue 1.

If a collection of graphs $\left\{G_{1}, \ldots, G_{p}\right\}$ are jointly connected and occurs infinitely often, it is proved [13] that their corresponding Perron matrices satisfy $\bigcap_{i=1}^{p} \mathcal{H}\left(P_{i}\right)=$ $\operatorname{span}\{\mathbf{1}\}=\mathcal{H}\left(\frac{1}{p} \sum_{i=1}^{p} P_{i}\right)=\bigcap_{i=1}^{r} \mathcal{H}\left(P_{i}\right)$ for the set of all communication graphs $\left\{G_{1}, \ldots, G_{r}\right\}$, where $r \geq p$.

Denote $\bar{P}=\frac{1}{p} \sum_{i=1}^{p} P_{i}$, we know $\bar{P}$ is the primitive Perron matrix of a strongly connected graph, and $\bar{P}$ has $\mathbf{1}$ as the right eigenvector associated with the simple eigenvalue 1 . We decompose the generalized eigenspace $\mathbb{E}^{n}$ of $\bar{P}$ as $E_{1} \oplus E_{s}$ [16], where $E_{1}=\operatorname{span}\{\mathbf{1}\}$ associated with the simple eigenvalue 1 , and $E_{s}$ is the collection of all the other eigenspaces. For any initial value $x(0)$, we decompose $x(0)=u(0)+w(0)$, where $u(0) \in E_{1}$ and $w(0) \in$ $E_{s}$. Because $u(0) \in \bigcap_{i=1}^{r} \mathcal{H}\left(P_{i}\right)$, the sequence given by $u(k+1)=P_{i(k)} u(k)$ is constant, and the limit $u^{*}=u(0)$. On the other hand, because $E_{s}$ is invariant under all $P_{i(k)}$ [16], the sequence of vectors given by $w(k+1)=P_{i(k)} w(k)$ all belong to $E_{s}$ which leads to the limit $w^{*} \in E_{s}$. From the properties of the infinite paracontracting process [15], $w^{*}$ has to be in the fixed subspace of $\bigcap_{i=1}^{r} \mathcal{H}\left(P_{i}\right)$. It means $w^{*} \in \operatorname{span}\{\mathbf{1}\}=E_{1}$, which yields $w^{*}=0$, because $E_{1} \cap E_{s}=\varnothing$. Therefore, the iterative process (18) has the limit $x^{*}=u^{*}$, and $u^{*}$ is given by the spectral projection of matrix $\bar{P}$, associated with the simple eigenvalue 1 , as [17] $x^{*}=u^{*}=\frac{\mathbf{1} \sigma^{T}}{\sigma^{T} \mathbf{1}} x(0)=\frac{\sum_{i=1}^{n} \sigma_{i} x_{i}(0)}{\sum_{i=1}^{n} \sigma_{i}} \mathbf{1}$, where $\sigma=$ $\left[\sigma_{1}, \ldots, \sigma_{n}\right]^{T}$ and $\sigma_{i}$ is the element of the diagonal matrix $\Sigma$. This finishes the proof for Theorem 1 .

\section{B. Proof of Corollary 1}

According to [18], balanced graph Laplacian matrix has $\mathbf{1}^{T}$ and $\mathbf{1}$ as the left and right eigenvectors associated with the eigenvalue zero, respectively. Therefore, every associated $P_{i(k)}$ has $\sigma^{T}=\left[\sigma_{1}, \ldots, \sigma_{n}\right]$ and $\mathbf{1}$ as the left and right eigenvectors, respectively, associated with the eigenvalue 1. With this property, the proof of Theorem 1 applies to Corollary 1. This finishes the proof of Corollary 1.

\section{REFERENCES}

[1] J. Mitola, An integrated agent architecture for software defined radio. $\mathrm{PhD}$ thesis, The Royal Institute of Technology, KTH, 2000.

[2] I. F. Akyildiz, B. F. Lo, and R. Balakrishnan, "Cooperative spectrum sensing in cognitive radio networks: A survey," Physical Communication, vol. 4, no. 1, pp. $40-62,2011$.

[3] K. Ben Letaief and W. Zhang, "Cooperative communications for cognitive radio networks," Proceedings of the IEEE, vol. 97, pp. 878 -893 , May 2009.

[4] D. Cabric, S. Mishra, and R. Brodersen, "Implementation issues in spectrum sensing for cognitive radios," Conference Record of the Thirty-Eighth Asilomar Conference on Signals, Systems and Computers, vol. 1, pp. $772-776,2004$.

[5] A. Ghasemi and E. Sousa, "Collaborative spectrum sensing for opportunistic access in fading environments," First IEEE International Symposium on New Frontiers in Dynamic Spectrum Access Networks, DySPAN 2005., pp. $131-136,2005$.

[6] J. Ma and Y. Li, "Soft combination and detection for cooperative spectrum sensing in cognitive radio networks," IEEE Global Telecommunications Conference, pp. 3139 -3143, 2007.

[7] F. E. Visser, G. J. Janssen, and P. Pawelczak, "Multinode spectrum sensing based on energy detection for dynamic spectrum access," IEEE Vehicular Technology Conference, pp. 1394 - 1398, 2008.

[8] Z. Li, F. R. Yu, and M. Huang, "A distributed consensus-based cooperative spectrum-sensing scheme in cognitive radio," IEEE Transaction on Vehicular Technology, vol. 59, pp. 383-393, Jan 2010.

[9] M. A. F.F. Digham and M. K. Simon, "On the energy detection of unknown signals over fading channels," IEEE Transactions on Communications, vol. 55, pp. 21-24, 2007.

[10] V. Kostylev, "Energy detection of a signal with random amplitude," in IEEE International Conference on Communications, vol. 3, pp. 16061610, 2002.

[11] R. Olfati-Saber, J. Fax, and R. Murray, "Consensus and cooperation in networked multi-agent systems," Proceedings of the IEEE, vol. 95, pp. $215-233$, Jan. 2007.

[12] S. Patterson, B. Bamieh, and A. El Abbadi, "Convergence rates of distributed average consensus with stochastic link failures," IEEE Transactions on Automatic Control, vol. 55, no. 4, pp. 880 -892, 2010.

[13] L. Xiao, S. Boyd, and S. Lall, "A scheme for robust distributed sensor fusion based on average consensus," Proc. 4th Int. Symp. Inf. Process. Sens. Netw., pp. 63-70, 2005.

[14] Z. Qu, Cooperative Control of Dynamical Systems, Application to Autonomous Vehicles. Springer-Verlag London, 2009.

[15] L. Elsner, I. Koltracht, and M. Neumann, "On the convergence of asynchronous paracontractions with applications to tomographic reconstruction from incomplete data," Linear Algebra Appl., vol. 130, pp. 65-82, 1990.

[16] J. D. Meiss, Differential Dynamical Systems. SIAM Mathematical Modeling and Computation.

[17] M. Carl, Matrix Analysis and Applied Linear Algebra. SIAM, 2000.

[18] R. Olfati-Saber and R. Murray, "Consensus problems in networks of agents with switching topology and time-delays," IEEE Transactions on Automatic Control, vol. 49, no. 9, pp. 1520 - 1533, 2004. 